\title{
Exploring the Mechanical Properties of Single Vimentin Intermediate Filaments by Atomic Force Microscopy
}

\author{
C. Guzmán ${ }^{1 *}$, S. Jeney ${ }^{1}$, L. Kreplak ${ }^{2}$, S. Kasas ${ }^{3,4}$, A. J. Kulik ${ }^{1}$ \\ U. Aebi ${ }^{2}$ and L. Forró ${ }^{1}$
}

${ }^{1}$ Institut de Physique de la Matière Complexe, Ecole Polytechnique Fédérale de Lausanne (EPFL), CH-1015 Lausanne, Switzerland

${ }^{2}$ M. E. Müller Institute for Structural Biology, Biozentrum University of Basel

Klingelbergstr. 704056

Basel, Switzerland

${ }^{3}$ Institut de Neurosciences

EPFL, CH-1015

Lausanne, Switzerland

${ }^{4}$ Institut de Biologie Cellulaire et de Morphologie, UNIL CH-1005 Lausanne

Switzerland
Intermediate filaments (IFs), together with actin filaments and microtubules, compose the cytoskeleton. Among other functions, IFs impart mechanical stability to cells when exposed to mechanical stress and act as a support when the other cytoskeletal filaments cannot keep the structural integrity of the cells. Here we present a study on the bending properties of single vimentin IFs in which we used an atomic force microscopy (AFM) tip to elastically deform single filaments hanging over a porous membrane. We obtained a value for the bending modulus of non-stabilized IFs between $300 \mathrm{MPa}$ and $400 \mathrm{MPa}$. Our results together with previous ones suggest that IFs present axial sliding between their constitutive building blocks and therefore have a bending modulus that depends on the filament length. Measurements of glutaraldehyde-stabilized filaments were also performed to reduce the axial sliding between subunits and therefore provide a lower limit estimate of the Young's modulus of the filaments. The results show an increment of two to three times in the bending modulus for the stabilized IFs with respect to the non-stabilized ones, suggesting that the Young's modulus of vimentin IFs should be around $900 \mathrm{MPa}$ or higher.

(C) 2006 Elsevier Ltd. All rights reserved.

Keywords: vimentin; intermediate filaments; atomic force microscopy; bending stiffness; axial sliding between dimmers; glutaraldehyde-stabilized filaments
The superfamily of IF proteins, which includes at least 65 distinct proteins in humans, ${ }^{2}$ has been divided into five different types according to their primary structure, gene structure, assembly properties and their tissue-specific expression patterns regulated during development. ${ }^{1}$

Structural studies have identified a common building block for all cytoplasmic IFs consisting of a very elongated rod-like dimer $(45-50 \mathrm{~nm})$ based on an $\alpha$-helical coiled-coil structure. ${ }^{7}$ This rod-like dimer exhibits a "tripartite" structure formed by an $\alpha$-helical central rod domain and flanked by non$\alpha$-helical head and tail domains. The central rod is composed of four consecutive $\alpha$-helical segments $1 \mathrm{~A}, 1 \mathrm{~B}, 2 \mathrm{~A}$ and $2 \mathrm{~B}$ that harbor a pronounced sevenresidue periodicity, called heptad repeat, in the distribution of hydrophobic residues, ${ }^{7}$ exhibiting the signature of a coiled-coil structure. The four $\alpha-$ helical segments are interconnected by short, variable linkers L1, L12 and L2 ${ }^{7}$ and have a structure and length that is highly conserved in vertebrate cytoplasmic IFs. ${ }^{1}$ In contrast, the length and
Abbreviations used: IF, intermediate filament; AFM, atomic force microscopy; ULF, unit-length filaments; MT, microtubule; TEM, transmission electron microscopy.

E-mail address of the corresponding author: camilo.guzman@epfl.ch 
sequence of the head and tail domains are extremely variable giving the IF superfamily its great diversity. ${ }^{1}$ The elongated, rod-like IF dimer can, under appropriate buffer conditions, self-assemble in vitro into $10 \mathrm{~nm}$ wide filaments ${ }^{8}$ (see Figure 1).

Here we focus on vimentin, a type III IF protein expressed abundantly in fibroblasts and endothelial cells. Vimentin IFs have been proposed as the dominant cytoskeletal elements in determining the mechanical stability of fibroblasts ${ }^{3}$ as well as the rigidity of circulating lymphocytes. ${ }^{9,10}$ The vimentin assembly process (assembly group 2 according to the classification by Strelkov et al..$^{7}$ ) starts from an antiparallel lateral assembly of two dimers into halfstaggered tetramers that further associate laterally to form full-width unit-length filaments (ULFs). The ULFs, then, anneal longitudinally into immature irregular filaments that radially compact to eventually yield mature filaments of proper length and width. ${ }^{2}$

Knowledge about the IF's mechanical properties and their functional role in cells has increased in the last few years thanks to a series of studies mainly performed at the filament network ${ }^{11-14}$ and bundle levels. ${ }^{15,16}$ The only studies at the single filament level were done by Mücke, Kreplak, and coworkers. ${ }^{17,18}$ Mücke and colleagues used imaging by atomic force microscopy (AFM) to measure the apparent persistence length, $\lambda$, of vimentin IFs. They estimated the value for $\lambda$ to be on the order of $1 \mu \mathrm{m}$ by making different assumptions concerning the adsorption mechanism of IFs on a number of different solid supports used. ${ }^{17}$ In their experiment, Kreplak and colleagues used an AFM tip to stretch single desmin, keratin or neurofilaments, adsorbed to a solid support, up to their breaking limit. ${ }^{18}$ This experimental approach suffers from the limitation that the lateral force applied is not controlled and

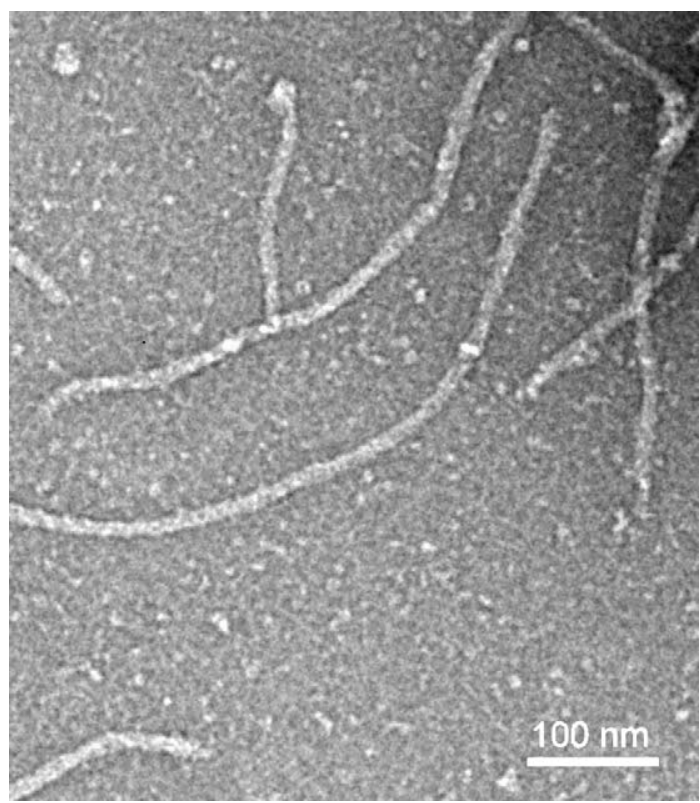

Figure 1. A TEM image of in vitro assembled hamster vimentin intermediate filaments. therefore yielding a description of the magnitude of the stretching without quantifying the applied forces.

In the previous studies mentioned above there is one missing parameter that helps to describe the mechanical properties of IFs, and that is essential to quantify their properties at the network and bundle levels. This parameter is the bending stiffness ( $\left.E_{\text {Bending }}\right)$ representing the resistance of a tube upon bending thereby determining its behavior under such kind of deformation. Theoretically the persistence length and the bending modulus are related by the expression:

$$
E_{\text {Bending } I}=k_{B} T \lambda
$$

where:

$$
I=\frac{\pi\left(D_{\text {Ext }}^{4}-D_{\text {Int }}^{4}\right)}{64}
$$

is the second moment of area, $k_{B}$ the Boltzmann constant, and $T$ the absolute temperature. $D_{\text {Ext }}$ and $D_{\text {Int }}$ are the outer and inner diameters of the tube. In the particular case of isotropic materials, i.e. materials that exhibit the same mechanical properties in all directions (i.e. no shearing between their building blocks), the bending stiffness corresponds to the Young's modulus ( $E_{\text {Young }}$. ${ }^{19}$

In order to rigorously measure the $E_{\text {Bending value of }}$ a single vimentin IF, we applied a method developed by Salvetat et al..$^{20}$ for the study of filamentous nanostructures. This method was successfully applied to another cytoskeletal filament moiety, to microtubules (MTs), ${ }^{21}$ as well as to biopolymers such as amyloid fibrils (Sheena Radford, University of Leeds UK and Cait MacPhee, University of Cambridge UK, personal communications). The principle of the technique is to span a single filament over a hole and image it by AFM with increasing forces and under physiological conditions. From the images it is possible to extract the deflection of the filament under a given applied force.

Most of the studies performed up to now on the mechanical properties of cytoskeletal filaments at the single filament level have employed crosslinking agents such as glutaraldehyde. ${ }^{21-24}$ In the case of vimentin IFs the filaments were sufficiently stable to be spanned over a $250 \mathrm{~nm}$ wide hole without the need of any stabilizing agent and this allowed us to test their properties under physiological conditions. In a second step, glutaraldehyde was used to reinforce the bonds between subunits to test the influence of intermolecular motion on $E_{\text {Bending. }}$. Calculating the magnitude of the bending modulus in the presence of glutaraldehyde would allow us to estimate a lower limit of the Young's modulus and thereby describe the effect of pure stretching.

\section{Theory}

In the same way as Salvetat et $a l .{ }^{20}$ did with carbon nanotubes, a single vimentin IF was modelled as a 
clamped beam that rests over a gap, i.e. a hole with a diameter $L \sim 250 \mathrm{~nm}$ in a porous substrate, and is being bent by pushing with an AFM tip. We applied a controlled force on the filament and registered the filament's deflection as a function of the applied force (see Figure 2). From the relationship:

$$
\delta=\frac{F L^{3}}{192 E_{\text {Bending } I}}
$$

we calculated the bending modulus ( $\left.E_{\text {Bending }}\right)$ of the filament. ${ }^{19}$ Here $\delta$ represents the deflection and $F$ the applied force (see equation (2)).

In the presence of shearing between subunits, equation (3) can be written as a combined contribution that involves the sliding between subunits and their stretching: ${ }^{19}$

$$
\begin{aligned}
\delta & =\delta_{\text {Stretching }}+\delta_{\text {Shearing }}=\frac{F L^{3}}{192 E_{\text {Young }} I}+f_{s} \frac{F L}{4 G A} \\
& =\frac{F L^{3}}{192 E_{\text {Bending }} I}
\end{aligned}
$$

and hence:

$$
\frac{1}{E_{\text {Bending }}}=\frac{1}{E_{\text {Young }}}+\frac{1}{G} \frac{3 f_{s}\left(D_{\text {Ext }}^{2}+D_{\text {Int }}^{2}\right)}{L^{2}}
$$

where $E_{\text {Young }}$ represents the Young's modulus that is equal to $E_{\text {Bending }}$ in the case of a long filament and $G$ is the shear modulus. A represents the crosssectional area and $f_{s}$ is a shape factor, equal to 1.1 for filled cylinders and 1.38 for hollow ones. ${ }^{19}$

\section{Results}

AFM images revealed filaments that remained well attached to both sides of the hole thus allowing us to push the filament with the AFM

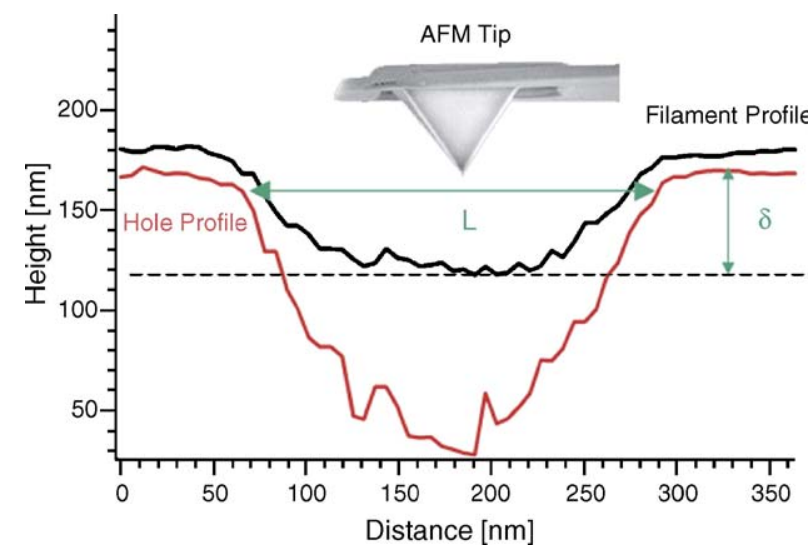

Figure 2. Principle of the AFM experiment for determining the bending modulus of an IF. The AFM tip pushes the IF into the hole and from the height difference between the IF's lowest point (maximal deflection) and the flat substrate around the hole, we calculated the deflection. Note that in this cartoon the AFM tip is not drawn to scale with respect to the profile dimensions. tip and bend it without making it slide into the hole (see Figure 3).

Deflection versus force plots (see Figure 4) show that within a force range of $0.06 \mathrm{nN}$ to $0.13 \mathrm{nN}$ the filaments exhibited an elastic behavior, meaning that after decreasing the applied force they fully recovered from the force-induced deflection. Errors in the graph for each measurement point (see Figures 4 and 5) stem from the variation in the series of images recorded of the same filament with the same force applied $(\geq 4$ images per point).

By estimating the length of the filaments for the two extreme forces applied, it is possible to determine that their length has increased by at least $10 \%$ at the maximum elastic force $(0.13 \mathrm{nN})$ applied. This stretching was achieved with a negligible slippage of the filament within the hole (see Materials and Methods). Moreover, if we calculate the applied stress at the contact area (i.e. applied force over contact area), we can see that IFs are elastic up to $\sim 0.4 \mathrm{MPa}$ of applied stress.

From equation (3) we obtained the bending modulus for several unfixed vimentin IFs: their value ranged from $300 \mathrm{MPa}$ to $400 \mathrm{MPa}$, being consistent for different filaments (see Figure 5). In each case, the results are subject to an error of $50 \%$, the main source residing in an uncertainty of $\sim 15 \%$ in determining the diameter of the hole L. Note that in equation (3), the deflection $\delta$ depends on $L$ by the third order thus propagating the $\sim 15 \%$ error into a $\sim 50 \%$ error.

It is also important to mention that the number of subfilaments and their packing within the IFs would change the second moment of area in our clamped beam model and thereby affect the final result of the bending modulus. To test this, we performed calculations with the second moment of area for different internal configurations such as a tube (i.e. with $10 \mathrm{~nm}$ outer and $3 \mathrm{~nm}$ inner diameter ${ }^{25}$ ) and a solid $10 \mathrm{~nm}$ diameter cylinder, ${ }^{26}$ finding negligible differences in magnitude $(\sim 2 \%)$ when compared to the $50 \%$ error that we already had.

Furthermore, we modelled the bending modulus as a function of the number of subunits ( $n$ ) per filament cross-section. Accordingly, as we increase this number, the bending modulus approaches the value obtained for solid cylinder. Interestingly, the bending modulus does not follow the apparent $1 / n$ dependency suggested by Fudge et al. ${ }^{16}$

Last but not least, we calculated the bending modulus of IFs stabilized by cross-linking with $0.5 \%$ (v/v) glutaraldehyde to be $\sim 900 \mathrm{MPa}$. Not only does this result yield a two to three times higher value compared to unfixed filaments (see Figure 6), but it also prompted us to think that adjacent subfilaments may slide relative to one another within an IF. The extra covalent inter-subunit bonds created by the glutaraldehyde reduce the amount of sliding (by increasing the shear modulus $G)$ and thus produce an increase in the bending modulus $E_{\text {Bending }}$ (see Figure 7 ). In the case where there would be no sliding but only stretching of the 

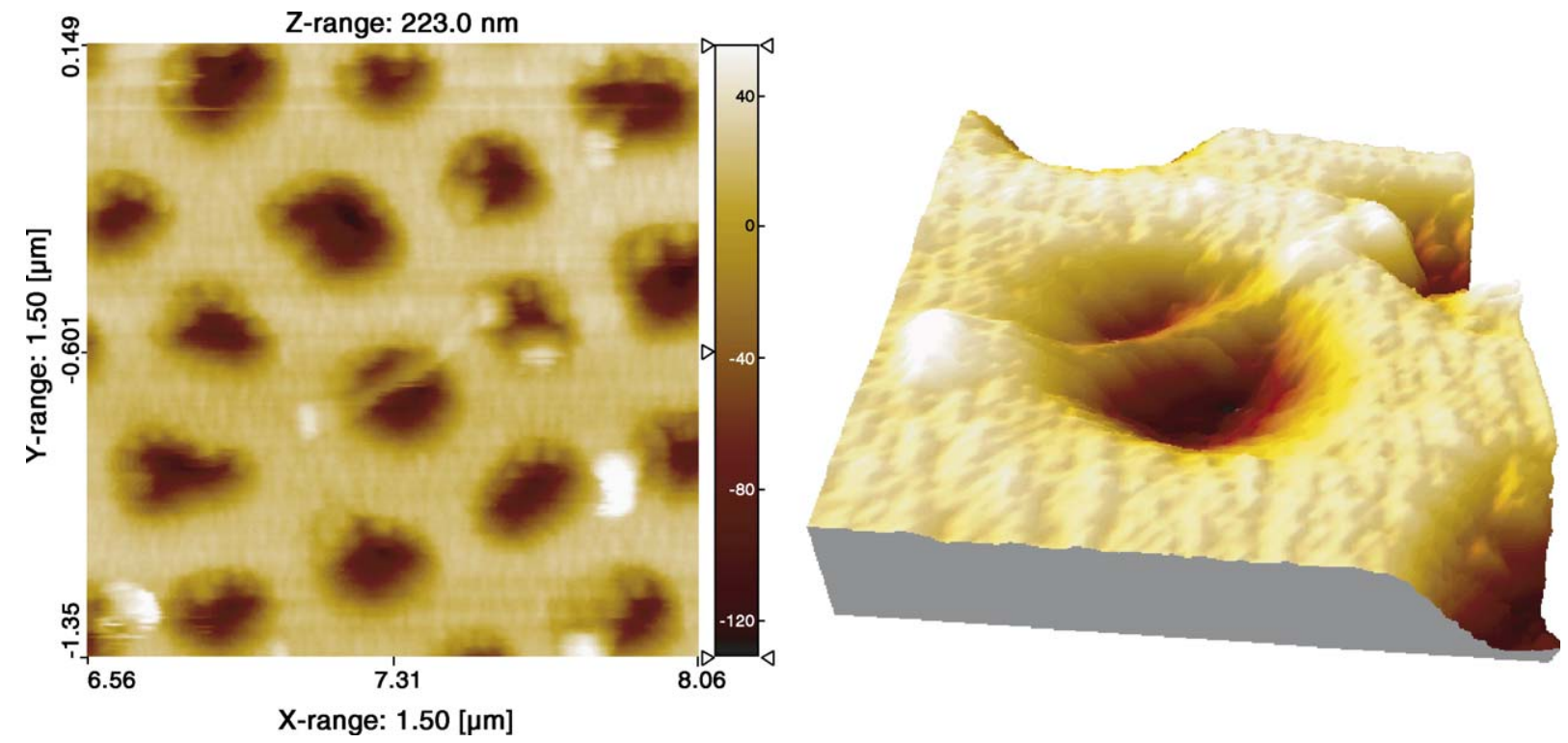

Figure 3. Left: AFM image of a single vimentin IF resting over a hole in a substrate of aluminum oxide. The scan size is $1.5 \mu \mathrm{m}$ and the applied force $0.11 \mathrm{nN}$. Right: 3-D representation of the same filament.

subunits within the filament, the bending modulus would equal the Young's modulus.

\section{Discussion}

\section{Mechanical properties of single vimentin IFs at the nanoscale}

IFs can readily adsorb to a wide range of solid supports and imaged by AFM in physiological buffer. ${ }^{27}$ Here, we have extended this approach by imaging single vimentin filaments spanning over a $250 \mathrm{~nm}$ wide hole on a polished alumina membrane. Due to the constant force applied by the AFM tip

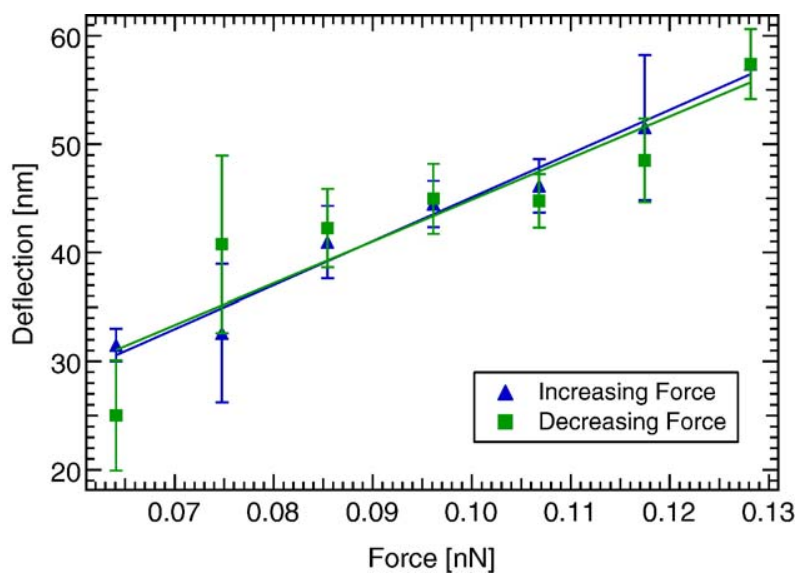

Figure 4. Example of the deflection of one IF as a function of the applied force ( $E_{\text {Bending }}=300 \pm 150 \mathrm{Mpa}$, determined from the slope of the linear fit according to equation (4)). In the graph it is possible to see that within this range of forces the filament is elastic (i.e. it returns to its original position after the force is decreased). during scanning, native (i.e. unstabilized) filaments were bending reversibly with an apparent stiffness of $\sim 300 \mathrm{MPa}$. The maximum bending observed corresponded to a filament stretching of $\sim 10 \%$. In the geometry used in this study, the measured bending stiffness is a complex value that depends on three parameters: the Young's modulus, the shear modulus and the length of the filament (see equation (5)). In the case of IFs, the shear modulus cannot be neglected because the filament is built by the longitudinal and lateral interaction of $\sim 45 \mathrm{~nm}$ long rods that have the potential to slide longitudinally past each other. ${ }^{15,17,28}$ Hence, a cross-linker such as glutaraldehyde is able to create covalent bonds between adjacent dimer rods that should reduce the amount of sliding. As expected from equation (5), glutaraldehyde-fixed IFs exhibit a significantly higher bending stiffness of $\sim 900 \mathrm{MPa}$ that, to first

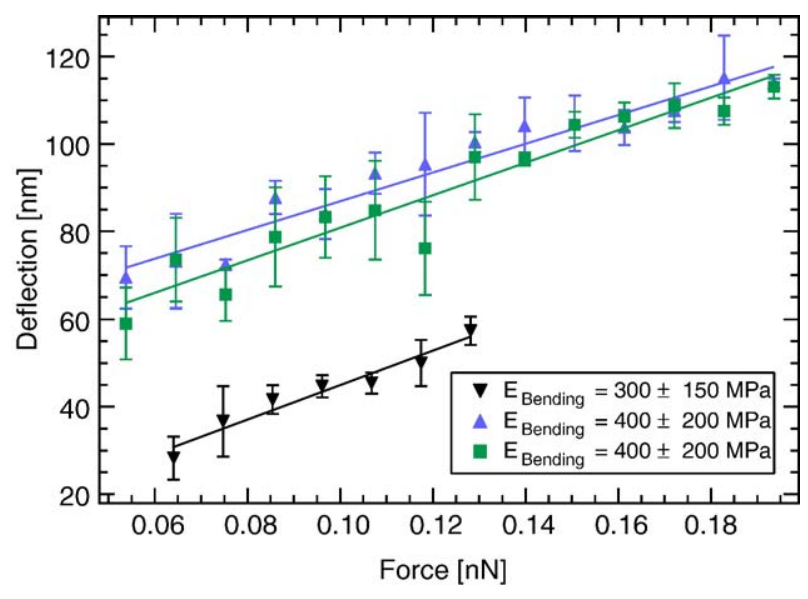

Figure 5. Deflection versus applied force showing the plot of three different unfixed IFs with their respective bending modulus ( $\left.E_{\text {Bending }}\right)$. 


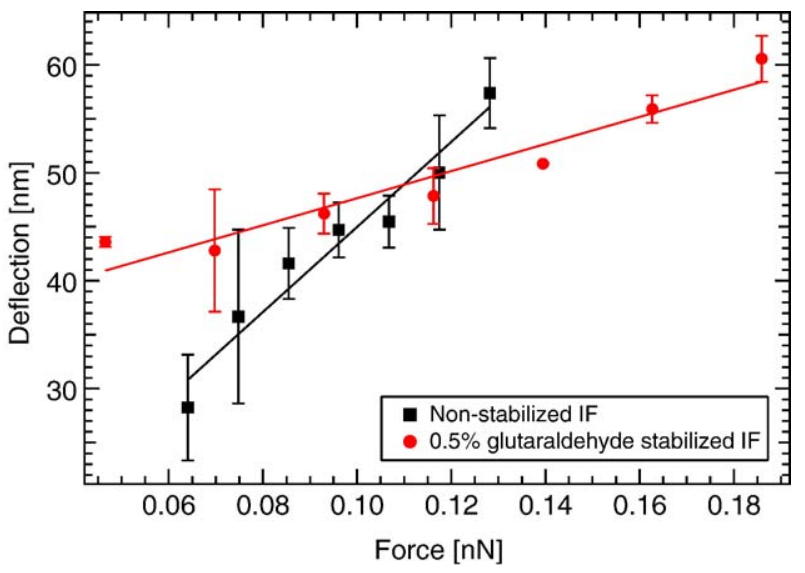

Figure 6. Comparison between a non-stabilized IF ( $E_{\text {Bending }}=300( \pm 150) \mathrm{MPa}$ ) and an IF stabilized by crosslinking with $0.5 \%$ glutaraldehyde $\left(E_{\text {Bending }}=900 \pm 450\right.$ $\mathrm{MPa})$. As a consequence, the bending modulus increases by a factor of 3 upon stabilization of the filaments.

approximation, can be considered as a lower value of the Young's modulus ( $\left.E_{\text {Young }}\right)$. Then, assuming a Young's modulus of $900 \mathrm{MPa}$, we can estimate from Figure 7 an upper value for the shear modulus $G=2.4 \mathrm{MPa}$. This appears to be a reasonable estimate, since Kis et al. have measured a shear modulus of $1.4 \mathrm{MPa}$ for glutaraldehyde-fixed microtubules with exactly the same experimental approach. ${ }^{21}$

It is important to notice that in our case the Young's modulus is several orders of magnitude higher than the shear modulus, being indicative of a strong anisotropic behavior. Hence, mechanical data on IFs have to be compared while keeping in mind the geometry and the scale of the different experiments. It has been demonstrated recently that vimentin IFs have a persistence length of $\sim 1 \mu \mathrm{m}$, corresponding to a bending modulus of $\sim 8 \mathrm{MPa}$ (see equation (1)). ${ }^{17}$ This persistence length was obtained by adsorbing filaments on different supports without any applied force and in the presence of a high interaction between the support and the filaments. According to our data, in this case the bending behavior should be dominated by longitudinal shearing of the subunits within the filaments as proposed by Mücke et al. ${ }^{17}$ Hence, we can combine our measurements and theirs to investigate more systematically the anisotropic behavior of IFs leading to a length dependence on the bending stiffness. Such anisotropic behavior has already been observed with microtubules. ${ }^{21,29}$ Also, it should be noted that the measurements by Mücke et al. ${ }^{17}$ were done on several $\mu \mathrm{m}$ long IFs whereas ours were performed on short filaments spanning a $\sim 250 \mathrm{~nm}$ hole, i.e. only being about $25 \%$ of the filament's persistence length.

A value of 7-8 $\mathrm{MPa}$ was also obtained for the Young's modulus of Hagfish slime threads that were stretched in sea water. ${ }^{16}$ These hydrated threads are bundles of approximately parallel, keratin-like IFs. Therefore, the first event happening upon stretching is most likely a better alignment of the filaments due to sliding relative to each other. We propose that Fudge et al. ${ }^{16}$ did not really measure a Young's modulus for the keratin IFs but rather the stiffness of a complex aggregation of filaments that can slide relative to one another and thereby give the impression that IFs are more compliant than they actually are. In order to obtain an estimate of the Young's modulus of IFs one can look at materials where the filaments are aligned and cross-linked to each other such as wool or hair. ${ }^{30}$ For these wet keratin fibres the Young's modulus is $\sim 2 \mathrm{GPa}$, which is comparable to the bending modulus that we measured for glutaraldehyde-fixed vimentin IFs.

Vimentin IFs cannot be considered as a homogeneous material. Within the filament cross-section, there is an average of 16 coiled-coil dimers, as determined by mass-per-length measurements. ${ }^{31}$ However, there are never 16 subfilaments depicted in an IF. Instead electron micrographs of unraveled filaments reveal the presence of four to eight subfilaments that coil around each other. ${ }^{32}$ These subfilaments, in turn, are made of either tetramers or octamers, i.e. two or four aligned coiled-coil dimers that interact laterally with each other. ${ }^{7}$ Most likely these subfilaments can moderately slide past each other longitudinally, thus yielding a relatively small shear modulus. To understand how the subfilaments can give rise to a large bending modulus, one has to realize that a single coiledcoil yields a bending modulus of $130( \pm 50) \mathrm{MPa}$. This is estimated from equation (1) using the persistence length measured for a single myosin molecule, i.e. $25( \pm 10) \mathrm{nm}^{33}$ and a coiled-coil diameter of $2 \mathrm{~nm}$. With this value in mind, it is easy to see that subfilaments, being either tetramers or octamers, should present a bending modulus of several hundreds $\mathrm{MPa}$.

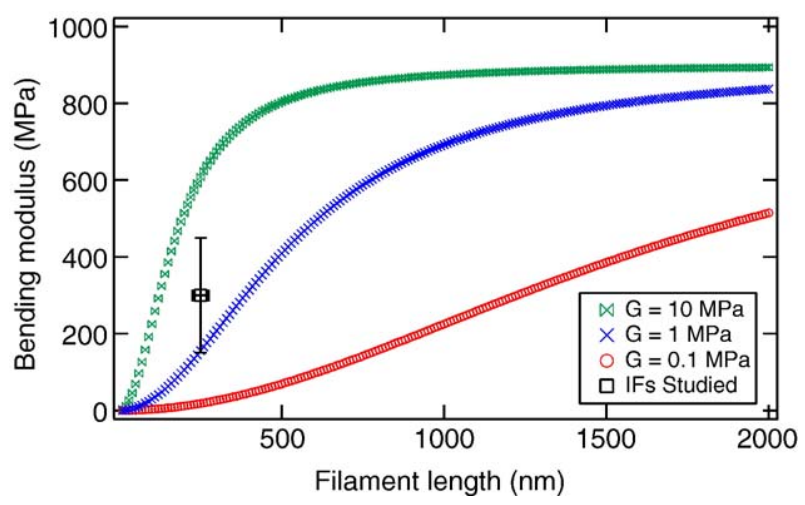

Figure 7. Plot of equation (5) displaying the behavior of $E_{\text {Bending }}$ as a function of filament length. The different curves represent different shear modulus values. For comparison, microtubules exhibit a shear modulus value $\mathrm{G}=1.4 \mathrm{MPa}$, as determined by Kis et al. ${ }^{21}$ The Young's modulus was chosen to be $900 \mathrm{MPa}$ for this plot. 


\section{IFs and the cytoskeleton}

The current cell mechanics approaches cannot easily separate the contributions of the actin filaments, microtubules and IFs networks to the stiffness of the cytoskeleton. In vitro rheological data on gels of the different filaments clearly show that actin gels are stiffer at low strain compared to microtubules and IFs. ${ }^{11}$ However, it is difficult to extract from these rheological data the contribution arising from the mechanical properties of single filaments. $^{34,35}$ This is where our experimental approach is offering new insights, since we are measuring directly the bending modulus of single filaments. In that respect, comparison between IFs and microtubules bending stiffness is interesting. Electron microscopy observations are in favor of microtubules exhibiting a much larger bending stiffness than IFs. While, this is true for a micrometer long IF, when a bending stress is applied locally over a short length $\sim 250 \mathrm{~nm}$ then vimentin IFs reveal a five times higher bending modulus. ${ }^{21}$ Hence, even if the vimentin IF network does not seem to strongly determine the global mechanical properties of the cytoskeleton at small strain, ${ }^{36}$ and that due to the higher radius of MTs $(25 \mathrm{~nm})$ with respect to the one of IFs $(10 \mathrm{~nm})$, the bending stiffness of MTs is still higher than the one of IFs; our data still indicate that IFs assemblies can be stiff enough to be involve in localized mechanotransduction events as expected from, for example, their binding to focal adhesion sites via the integrin complex. ${ }^{37}$

\section{Materials and Methods}

\section{IF polymerization}

A total of $50 \mu \mathrm{g}$ of recombinant Syrian hamster vimentin protein ( $>90 \%$ pure from Cytoskeleton Inc., USA) was diluted in vimentin subunit buffer (5 mM Pipes ( $\mathrm{pH} 7.0$ ), $1 \mathrm{mM}$ DTT) and transferred to the sample carrier of our spectrophotometer to control polymerization. The polymerization buffer (5 mM Pipes (pH 7.0), 1 mM DTT, 1.5 M $\mathrm{NaCl}$ ) was added to achieve a final protein concentration of $0.25 \mathrm{mg} / \mathrm{ml}$ in $150 \mathrm{mM} \mathrm{NaCl}$. The spectrophotometer temperature was kept at $35{ }^{\circ} \mathrm{C}$ to allow polymerization of filaments. The UV absorbance of the sample at wavelengths of $260 \mathrm{~nm}$ and $280 \mathrm{~nm}$ was recorded every $15 \mathrm{~s}$ for more than $1 \mathrm{~h}$.

After polymerization, the material was divided in two equal volumes. Both aliquots were diluted 20 times in subunit buffer and one of them was cross-linked with $0.5 \%(\mathrm{v} / \mathrm{v})$ glutaraldehyde.

\section{Electron microscopy}

Transmission electron microscopy (TEM) images of fully polymerized IFs were recorded to check the quality of the filaments (Figure 1). For this, $10 \mu \mathrm{l}$ of glutaraldehyde-stabilized $(0.1 \%)$ vimentin IFs were adsorbed to a previously glow-discharged carbon grid and then negatively stained with $2 \%(\mathrm{w} / \mathrm{v})$ uranyl acetate. The sample was dried and images were taken in a transmission electron microscope (Philips CM 20, Netherlands).

\section{AFM imaging}

All AFM images were taken in contact mode and in buffer solution. For this purpose, $40 \mu \mathrm{l}$ of a solution containing assembled vimentin IFs were adsorbed to a previously polished alumina ultrafiltration membrane (Whatman, UK). The membrane was also treated with 3triethoxysilylpropylamine (APES) (following the procedure described ${ }^{38}$ for functionalized mica) in order to improve the adsorption of the IFs. After $10 \mathrm{~min}$ the sample was washed to remove unattached material. IFs were always kept in buffer solution.

The alumina membrane with adsorbed IFs was mounted into our custom-made AFM liquid cell to keep physiological conditions (buffer solution) and a constant temperature of $20{ }^{\circ} \mathrm{C}$ using a temperature controller (Lakeshore 330 Autotuning Temperature Control, USA). We used a commercial AFM (Park Scientific M5, USA) and silicon nitride sharpened cantilevers with a nominal spring constant of $0.01 \mathrm{~N} / \mathrm{m}$ (Veeco Microlevers, USA). The spring constant of the cantilevers was calibrated using their resonance frequency. ${ }^{39}$

To perform stable and controlled pushing of the filament, we acquired at least four complete AFM images of the filaments and the surrounding substrate at each specific force. The force was gradually increase after each set of images and decreased in the same way until returning to the starting force. The scanning frequency was kept below $0.7 \mathrm{~Hz}$ to avoid cutting the filaments and to keep a constant pushing of the filament at the selected force.

We chose filaments with a length below $1 \mu \mathrm{m}$ in order to keep the filament within the scan size of our image. This allowed us to verify that the ends were not moving upon bending and to control that the filament was being stretched and not pushed into the hole.

\section{Data analysis}

From the series of AFM images taken for each filament at each force, we determined the deflection of the filament. This was done by comparing the height difference between the hard substrate and the filament center (mostly corresponding to the pore center) where the maximal deflection at the applied force was measured (Figure 3).

From the deflection we calculated the bending modulus of the filaments using the clamp beam model introduced above. We performed a linear fit on the deflection versus force plots and the bending modulus was estimated from the slope according to equation (3).

\section{Acknowledgements}

We thank G. Beney (EPFL) for polishing the alumina membranes, A. Z. Stasiak (UNIL) and C. Dieker (EPFL) for help on TEM imaging. C. G. thanks B. M. Riederer (UNIL) for a helpful introduction to the process of IFs extraction and 
polymerization. This work is partially supported by the National Center for Competence in Research "Nanoscale Science" of the Swiss National Science Foundation. L. K. was supported by a grant from the Swiss Society for Research on Muscular Diseases awarded to U. A. and S. Strelkov.

\section{References}

1. Chang, L. \& Goldman, R. D. (2004). Intermediate filaments mediate cytoskeletal crosstalk. Nature Rev. Mol. Cell Biol. 5, 601-613.

2. Herrmann, H. \& Aebi, U. (2004). Intermediate filaments: molecular structure, assembly mechanism, and integration into functionally distinct intracellular scaffolds. Annu. Rev. Biochem. 73, 749-789.

3. Eckes, B., Dogic, D., Colucci-Guyon, E., Wang, N., Maniotis, A., Ingber, D. et al. (1998). Impaired mechanical stability, migration and contractile capacity in vimentin-deficient fibroblasts. J. Cell Sci. 111, 1897-1907.

4. Fuchs, E. \& Cleveland, D. W. (1998). A structural scaffolding of intermediate filaments in health and disease. Science, 279, 514-519.

5. Eckes, B., Colucci-Guyon, E., Smola, H., Nodder, S., Babinet, C., Krieg, T. \& Martin, P. (2000). Impaired wound healing in embryonic and adult mice lacking vimentin. J. Cell Sci. 113, 2455-2462.

6. Wong, P. \& Coulombe, P. A. (2003). Loss of keratin 6 (K6) proteins reveals a function for intermediate filaments during wound repair. J. Cell Biol. 163, 327-337.

7. Strelkov, S. V., Herrmann, H. \& Aebi, U. (2003). Molecular architecture of intermediate filaments. BioEssays, 25, 243-251.

8. Herrmann, H. \& Aebi, U. (1999). Intermediate filaments assembly: temperature sensitivity and polymorphism. Cell Mol. Life Sci. 55, 1416-1431.

9. Brown, M. J., Hallam, J. A., Colucci-Guyon, E. \& Shaw, S. (2001). Rigidity of circulating lymphocytes is primarily conferred by vimentin intermediate filaments. J. Immunol. 166, 6640-6646.

10. Nieminen, M., Henttinen, T., Merinen, M., MarttilaIchihara, F., Eriksson, J. E. \& Jalkanen, S. (2006). Vimentin function in lymphocyte adhesion and transcellular migration. Nature Cell Biol. 8, 156-162.

11. Janmey, P., Euteneuer, U., Tarub, P. \& Schliwa, M. (1991). Viscoelastic properties of vimentin compared with other filamentous biopolymer networks. J. Cell Biol. 113, 155-160.

12. Ma, L., Xu, J., Coulombe, P. A. \& Wirtz, D. (1999). Keratin filament suspensions show unique micromechanical properties. J. Biol. Chem. 274, 19145-19151.

13. Ma, L., Yamada, S., Wirtz, D. \& Coulombe, P. A. (2001). A 'hot-spot' mutation alters the mechanical properties of keratin filament networks. Nature Cell Biol. 3, 503-506.

14. Yamada, S., Wirtz, D. \& Coulombe, P. A. (2002). Pairwise assembly determines the intrinsic potential for self-organization and mechanical properties of keratin filaments. Mol. Biol. Cell, 13, 382-391.

15. Kreplak, L., Franbourg, A., Briki, F., Leroy, F., Dallé, D. \& Doucet, J. (2002). A new deformation model of hard $\alpha$-keratin fibers at the nanometer scale: implications for hard $\alpha$-keratin intermediate filament mechanical properties. Biophys. J. 82, 2265-2274.

16. Fudge, D. S., Gardner, K. H., Forsyth, T., Riekel, C. \&
Gosline, J. (2003). The mechanical properties of hydrated intermediate filaments: Insights from hagfish slime threads. Biophys. J. 85, 2015-2027.

17. Mücke, N., Kreplak, L., Kirmse, R., Wedig, T., Herrmann, H., Aebi, U. \& Langowski, J. (2004). Assessing the flexibility of intermediate filaments by atomic force microscopy. J. Mol. Biol. 335, 1241-1250.

18. Kreplak, L., Bär, H., Leterrier, J. F., Herrmann, H. \& Aebi, U. (2005). Exploring the mechanical behavior of single intermediate filaments. J. Mol. Biol. 354, 569-577.

19. Gere, J. M. \& Timoshenko, S. P. (1997). Mechanics of Materials 4th edit. PWS, Boston.

20. Salvetat, J.-P., Briggs, G. A. D., Bonard, J.-M., Bacsa, R. R., Kulik, A. J., Stöckli, T. et al. (1999). Elastic and shear moduli of single-walled carbon nanotube ropes. Phys. Rev. Letters, 82, 944-947.

21. Kis, A., Kasas, S., Babić, B., Kulik, A. J., Benoît, W., Briggs, G. A. D. et al. (2002). Nanomechanics of microtubules. Phys. Rev. Letters, 89, 248101.

22. Gittes, F., Mickey, B., Nettleton, J. \& Howard, J. (1993). Flexural rigidity of microtubules and actin filaments measured from thermal fluctuations in shape. J. Cell Biol. 120, 923-934.

23. Venier, P., Maggs, A. C., Carlier, M.-F. \& Pantaloni, D. (1994). Analysis of microtubule rigidity using hydrodynamic flow and thermal fluctuations. J. Biol. Chem. 269, 13353-13360.

24. Vinckier, A., Dumortier, C., Engelborghs, Y. \& Hellemans, L. (1996). Dynamical and mechanical study of immobilized microtubules with atomic force microscopy. J. Vac. Sci. Tech. B, 14, 1427-1431.

25. Watts, N. R., Jones, L. N., Cheng, N., Well, J. S., Parry, D. A. D. \& Steven, A. C. (2002). Cryo-electron microscopy of trichocyte hard $\alpha$-keratin intermediate filaments reveals a low-density core. J. Struct. Biol. 137, 109-118.

26. Er Rafik, M., Doucet, J. \& Briki, F. (2004). The intermediate filament architecture as determined by $X$-ray diffraction modeling of hard $\alpha$-keratin. Biophys. J. 86, 3893-3904.

27. Mücke, N., Kirmse, R., Wedig, T., Leterrier, J. F. \& Kreplak, L. (2005). Investigation of the morphology of intermediate filaments adsorbed to different solid supports. J. Struct. Biol. 150, 268-276.

28. Aebi, U., Häner, M., Troncoso, J., Eichner, R. \& Engel, A. (1988). Unifying principles in intermediate filament structure and assembly. Protoplasma, 145, 73-81.

29. Pampaloni, F., Lattanzi, G., Jonás, A., Surrey, T., Frey, E. \& Florin, E.-L. (submitted for publication). Elastic properties of grafted microtubules. Proc. Natl. Acad. Sci. U.S.A. e-print arXiv:q-bio/0503037.

30. Dobb, M. G. \& Rogers, G. E. (1967). Electron microscopy of fibrous keratins. Symp. Fibrous Proteins, 1, 267-278

31. Herrmann, H., Häner, M., Brettel, M., Müller, S. A., Goldie, K. N., Fedtke, B. et al. (1996). Structure and assembly properties of the intermediate filament protein vimentin: the role of its head, rod and tail domains. J. Mol. Biol. 264, 933-953.

32. Aebi, U., Fowler, W. E., Rew, P. \& Sun, T.-T. (1983). The fibrillar substructure of keratin filaments unraveled. J. Cell Biol. 97, 1131-1143.

33. Schwaiger, I., Sattler, C., Hostetter, D. R. \& Rief, M. (2002). The myosin coiled-coil is a truly elastic protein structure. Nature Mater. 1, 232-235.

34. Onck, P. R., Koeman, T., van Dillen, T. \& van der Giessen, E. (2005). Alternative explanation of stiffening in cross-linked semiflexible networks. Phys. Rev. Letters, 95, 178102-178104. 
35. Storm, C., Pastore, J. J., Mackintosh, F. C., Lubensky, T. C. \& Janmey, P. A. (2005). Nonlinear elasticity in biological gels. Nature, 435, 191-194.

36. Wang, N. \& Stamenovic, D. (2002). Mechanics of vimentin intermediate filaments. J. Mus. Res. Cell Motil. 23, 535-540.

37. Kreis, S., Schönfeld, H.-J., Melchior, C., Steiner, B. \& Kieffer, N. (2005). The intermediate filament protein vimentin binds specifically to a recombinant integrin $\alpha 2 / \beta 1$ cytoplasmic tail complex and co-localizes with native $\alpha 2 / \beta 1$ in endothelial cell focal adhesions. Exp. Cell Res. 305, 110-121.

38. Colton, R. J., Engel, A., Frommer, J. E., Gaub, H. E., Gewirth, A. A., Guckenberger, R. et al. (1998). Procedures in Scanning probe Microscopes. John Wiley \& Sons Ltd., West Sussex, England.

39. Cleveland, J. P., Manne, S., Bocek, D. \& Hansma, P. K. (1993). A nondestructive method for determining the spring constant of cantilevers for scanning force microscopy. Rev. Sci. Instrum. 64, 403-405.

Edited by M. Moody

(Received 14 March 2006; received in revised form 10 May 2006; accepted 11 May 2006) Available online 26 May 2006 